\title{
Clinical Evaluation of Noninvasive ECGI Epi-Endocardial Mapping Accuracy
}

\author{
Mikhail Chmelevsky ${ }^{1,2}$, Margarita Budanova ${ }^{1}$, Stepan Zubarev ${ }^{1}$, Danila Potyagaylo², \\ Tatjana Treshkur ${ }^{1}$, Dmitry Lebedev ${ }^{1}$ \\ ${ }^{1}$ Almazov National Medical Research Center, Saint-Petersburg, Russia \\ ${ }^{2}$ EP Solutions SA, Yverdon-les-Bains, Switzerland
}

\begin{abstract}
Although ECG imaging technology has been in development for many years, the clinical validation of epiendocardial ECGI mapping has been conducted only on relatively small groups of patients.

This study was performed to evaluate epi-endocardial ECGI mapping accuracy for the pacings from implanted pacemakers in a single center single-blind cross-sectional study. Thirty patients with previously implanted pacemakers underwent epi-endocardial ECGI mapping using "Amycard 01C EP Lab" system (EP Solutions SA, Switzerland).

The median (25-75\% IQR) geodesic distance between noninvasively reconstructed and the reference pacing sites was 8 (5-11) $\mathrm{mm}$ for the LV epicardial and 4 (2-6) $\mathrm{mm}$ for the $R V$ endocardial pacings.

This study showed sufficient accuracy of epi-endocarial ECGI technology to use it in a routine clinical practice for identification of focal arrhythmia sources.
\end{abstract}

\section{Introduction}

Topical diagnosis of focal arrhythmias using different invasive electroanatomic mapping systems is based on the registration of electrical potentials on the endo- or epicardial surface. At the same time, simultaneous registration of early activation zones on both epi- and endocardium remains a challenging task, especially in complex and unstable atrial and ventricular arrhythmias. Noninvasive ElectroCardioGraphic Imaging (ECGI) in combination with CT or MRI for obtaining precise heart and torso geometries is a method that allows to electrically map endocardial and epicardial surfaces. Although some available noninvasive ECGI systems are mapping the epicardial surface only, few studies have been performed to validate the accuracy of a novel epi-endocardial ECGI mapping technology $[1,2]$. Nevertheless, the lack of a detailed results description of the epi-/endocardial accuracy limits further integration of this technology into the clinical practice. Therefore, the aim of this work was to evaluate the accuracy of noninvasive ECGI epi- endocardial mapping based on the left ventricular (LV) epicardial and right ventricular (RV) endocardial pacings in patients with implanted CRT devices.

\section{Methods}

Thirty consecutive patients with previously implanted CRT devices and scheduled for regular check-ups and device optimization were included in our study. All patients underwent noninvasive ECGI mapping using Amycard 01C EP system (EP Solutions SA, Switzerland) (Figure 1). The study was reviewed and approved by the Ethical Committee of Almazov National Medical Research Center in Saint-Petersburg, Russia. Written informed consent was obtained from each patient after the detailed description and explanation of the study before the procedures. This single-center cross-sectional single-blind study was performed in accordance with the Good Clinical Practice guidelines and Helsinki declaration for biomedical research.

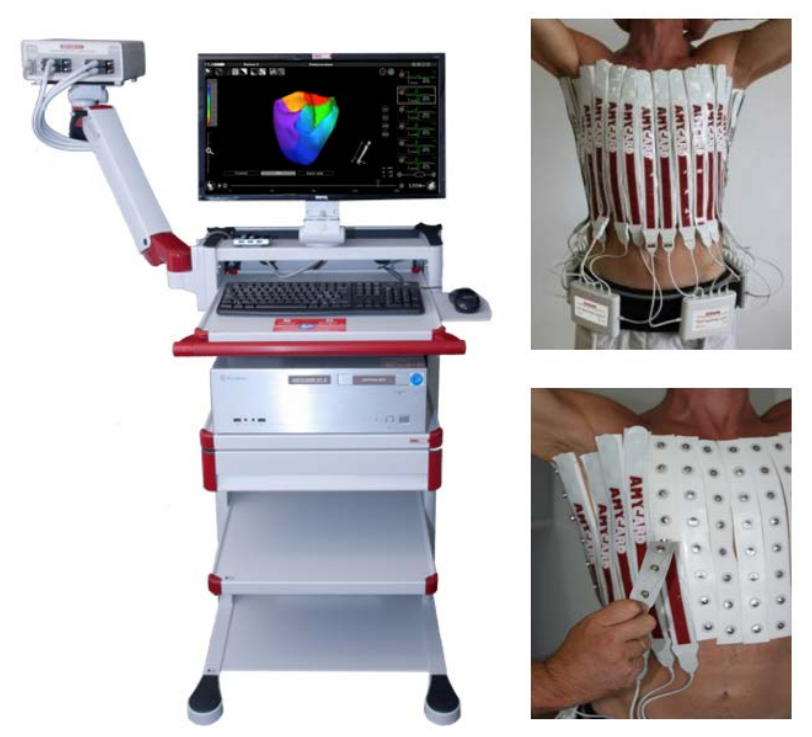

Figure 1. EP Solutions noninvasive ECGI mapping system 


\subsection{Multichannel ECG recording}

The overall methodology of ECGI with CT scan has been previously reported [3]. A total maximum number of 240 body surface electrodes were applied on the patient's torso and connected to the multichannel Amycard 01C EP system ECG amplifier. CRT device in each patient was programmed, and 10 min continuous ECGs of isolated $\mathrm{RV} / \mathrm{LV}$ pacing from implanted leads at rate not more than $90 \mathrm{bpm}$ were recorded during $10 \mathrm{sec}$ with breath hold applying a bandwidth of 0.05 to $500 \mathrm{~Hz}$, a sampling rate of 1000 samples/s, and an optional notch filter of $50 \mathrm{~Hz}$. After that, all original parameters of patient's CRT device were restored. The absence of fusion beats was considered as a criterion of the effective capture during RV/LV pacings. The parameters of the pacing amplitude and duration were selected individually for each patient based on the originally established parameters of the CRT device.

\subsection{Cardiac CT acquisition and image processing}

Directly after multichannel ECG recording all patients underwent cardiac CT imaging (Somatom Definition 128, Siemens AG, Germany) with applied body surface electrodes in order to obtain precise heart and torso anatomies. Afterwards, the obtained CT data was imported into Amycard 01C EP system software in DICOM format to segment and reconstruct a 3D voxel model of the torso and heart (Figure 2).

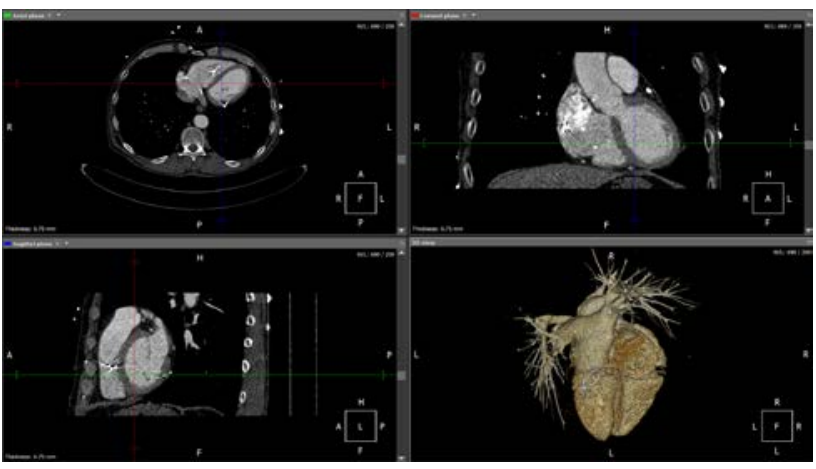

Figure 2. MPR and 3D voxel reconstruction.

A detailed 3D epi-endocardial ventricular polygon models were constructed based on the semi-automatic segmentation of CT data (Figure 3).

\subsection{Noninvasive epi-endocardial ECGI mapping}

An ECG fragment from the end of the pacing spike to the end of the T wave of the paced QRS complex was selected for the calculations. Epicardial and endocardial unipolar electrograms were reconstructed by EP Solutions inverse problem solution software using iterative Tikhonov regularization algorithm. Identification of early activation zone was determined based on a detailed visual analysis of isopotential maps with $1 \mathrm{~ms}$ step. Criteria for early activation zone were defined as follows: stable negative potential with radial (concentric) propagation along the heart surface appearing after beginning of QRS complex in II standard ECG lead, and with negative monophasic curve (QS) unipolar electrogram in the center of this zone. For the detected activation onsets, geodesic distances were computed to the pacing electrodes, whose locations were known exactly from the CT images.

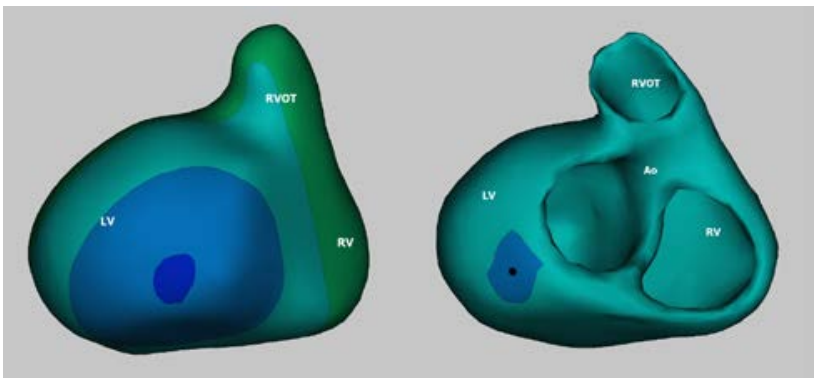

Figure 3. Epicardial (left) and epi-endocardial (right) polygon models. Isopotential map. Deep blue color shows an early activaton zone.

\section{Statistical analysis}

The distribution of obtained data was evaluated using Shapiro-Francia test with Royston's modification. Patient clinical characteristics and localization accuracy values of distances between real pacing points and early activation zones detected by ECGI were not normally distributed ( $\mathrm{p}$ $<0,001)$. Therefore, continuous variables were expressed as median (M) with min-max and $25-75 \%$ quartile values. Categorical data were presented as numbers and percentages. The Fisher exact and Pearson $\chi 2$ (with Yates' continuity correction) tests were used for the analysis of cross-tabulation tables of categorical data for clinical parameters. The Mann-Whitney U test was performed to compare independent samples, including patients' characteristics. The Wilcoxon signed-rank test was performed to compare two continuous variables of ECGI accuracy values calculated in the LV and RV. A P value $<0.01$ was considered as statistically significant due to Bonferroni correction. Statistical analysis was performed using Statistica v.12 (Statsoft Inc., USA) and MedCalc Statistical Software v.18.5 (MedCalc Software bvba, Ostend, Belgium). All data and comparisons were performed and presented in accordance to the Guidelines for reporting of observational studies (STROBE statement). 


\section{Results}

\subsection{Clinical Characteristics}

30 patients from 54 to 70 years (median 65; 25-75\% range 59-64; 8 male) with previously implanted CRT devices underwent ECGI with RV/LV isolated pacing. Among them nine patients had LBBB QRS morphology of the intrinsic rhythm, and seven of them had previous myocardial infarction. There was no significant association between clinical characteristics, pacing modalities, number of body surface electrodes and all ECGI accuracy values. The main patient's and ECGI characteristics are depicted in Table 1.

Table 1. Main patient's and ECGI characteristics

\begin{tabular}{lc}
\hline \multicolumn{1}{c}{ Characteristic } & Value \\
\hline Sex: [man], N (\%) & $23(76.7)$ \\
Age [years] & $63(46-73)$ \\
MI, N (\%) & $17(56.7)$ \\
LBBB, N (\%) & $24(80)$ \\
Body surface electrodes, N & $200(148-237)$ \\
\hline
\end{tabular}

Values are expressed as median (min-max) or as $N(\%)$. MI - myocardial infarction; LBBB - left bundle branch block.

\subsection{ECGI accuracy}

The median (25-75\% IQR) ECGI accuracy was 8 (5-11) $\mathrm{mm}$ in the LV and $4(2-6) \mathrm{mm}$ in the RV. The main ECGI accuracy values for both ventricles are presented in Table 2.

Table 2. Main characteristics of accuracy

\begin{tabular}{lll}
\hline \multicolumn{1}{c}{$\begin{array}{c}\text { Characteristic of } \\
\text { accuracy, mm }\end{array}$} & \multicolumn{1}{c}{ LV } & \multicolumn{1}{c}{ RV } \\
\hline$\%$ (N) of cases $\leq 5 \mathrm{~mm}$ & $27.6 \%(8)$ & $77.3 \%(22)$ \\
$\%$ (N) of cases $\leq 10 \mathrm{~mm}$ & $69.0 \%(20)$ & $86.7 \%(26)$ \\
Median & 8 & 4 \\
Low quartile (25\%) & 5 & 2 \\
Upper quartile (75\%) & 11 & 6 \\
Minimum & 2 & 1 \\
Maximum & 47 & 53 \\
\hline
\end{tabular}

There was no significant difference between LV and RV accuracy values ( $\mathrm{p}=0.023$ ). The accuracy distribution for $\mathrm{LV}$ and $\mathrm{RV}$ pacings is presented on the histograms and box-whisker plots in Figures 4, 5. Most of the pacing points have been detected with errors less than $10 \mathrm{~mm}(66,7 \%)$ in the $\mathrm{LV}$ and less than $5 \mathrm{~mm}$ (73.3\%) in the RV.

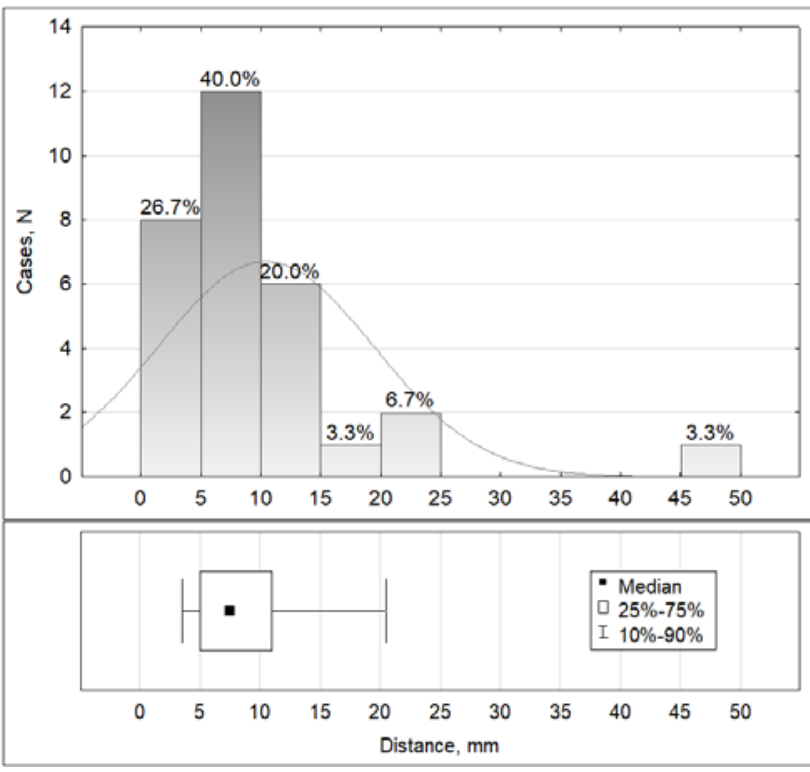

Figure 4. Histogram and Box-Whisker plot of the LV epicardial pacing accuracy. The fitted line represents a normal distribution.

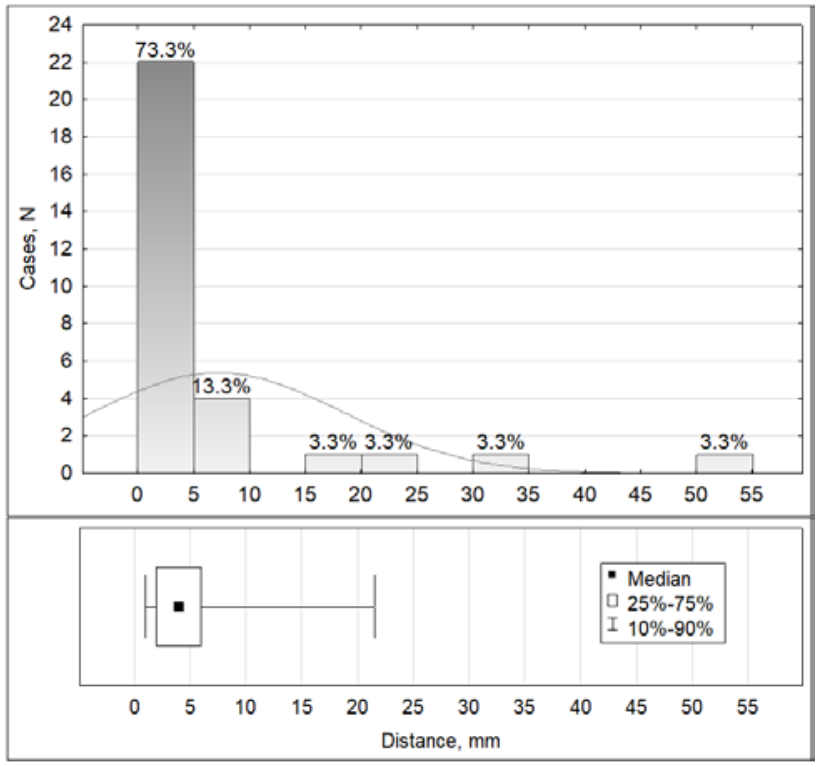

Figure 5. Histogram and Box-Whisker plot of RV endocardial pacing accuracy. The fitted line represents a normal distribution.

The 2D bag plot was used in order to evaluate an overall accuracy and visualize the location, spread and skewness of accuracy values in the RV and LV simultaneously (Figure 6). Tukey depth of accuracy was calculated as 9 $\mathrm{mm}$ for the LV epicardial pacing and $4 \mathrm{~mm}$ for the RV endocardial pacing. There was no significant correlation between $\mathrm{LV}$ and $\mathrm{RV}$ accuracy values. 


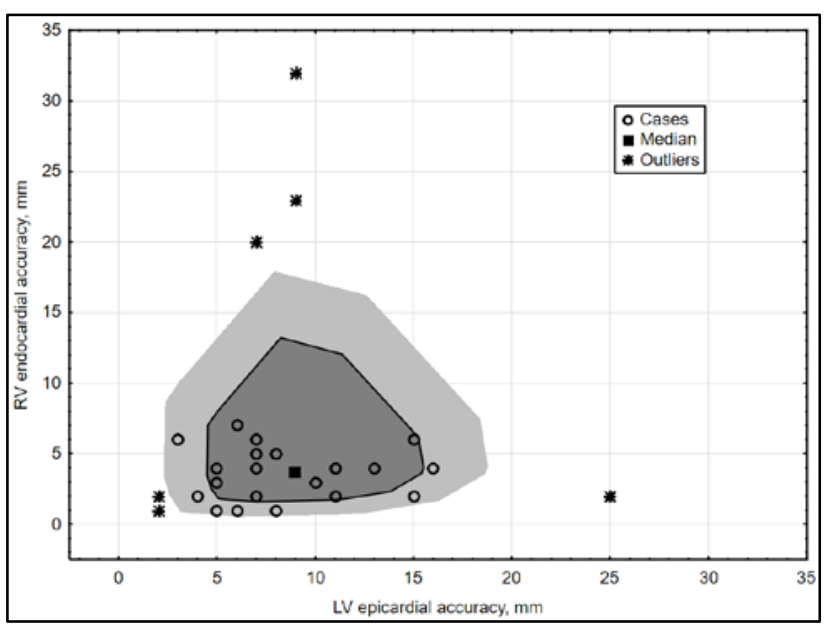

Figure 6. Bag plot of accuracy values. Bag (dark grey color) contains $50 \%$ of cases, fence (light grey) $-75 \%$ of cases.

\section{Discussion}

The main finding of this study was that the median accuracy of noninvasive ECGI mapping was $8 \mathrm{~mm}$ for the epicardial LV pacing and $4 \mathrm{~mm}$ for the RV endocardial pacing. Furthermore, this is the first single-center crosssectional single-blind study that specifically demonstrates noninvasive ECGI accuracy on epi- and endocardial surfaces using the commercial iterative Tikhonov regularization algorithm.

Previous studies reported the ECGI accuracy of approximately 10-15 mm on the epicardial surface using different algorithms $[4,5]$. At the same time, number of ECGI studies for the endocardial surface is limited despite the fact, that endocardium is more important for detecting and understanding of variety of arrhythmias.

The results of this study demonstrate accuracy less than $10 \mathrm{~mm}$, which is comparable to the tip of the navigation and ablation catheters used in the EP labs. A pretty narrow inter-quartile range shows that a majority of the pacing points was noninvasively detected with sufficient accuracy, and close to the median values. Moreover, the Tukey depth calculated on the bag plot as well as location and shape of the bag (50\% of cases) and looped fence demonstrate robustness of the utilized ECGI algorithm. Nevertheless, the lack of significant correlation between $\mathrm{RV}$ and $\mathrm{LV}$ pacings indicates that current algorithm can reconstruct unipolar electrograms unequally on the $R V$ and LV surfaces.

Another important finding is that the number of outliers is larger in the RV than in the LV. To explain this fact we have to take into account that in some patients $R V$ electrode was implanted in the septal area, which is probably an opaque region for the current noninvasive system.

\subsection{Study limitations}

Verification of accuracy using CRT devices is limited to the LV lateral wall, RV apex and septum, while other anatomical regions cannot be validated in the same way.

The presence of scar and fibrotic tissue in patients with previous MI violates the underlying mathematical model of the inverse calculations, which may lead to significant ECGI errors.

\section{Conclusions}

This study demonstrated a sufficient accuracy of noninvasive ECGI epi-endocardial mapping to detect early activation zones for focal arrhythmias on the epicardial LV lateral wall and endocardial RV apex making this beat-tobeat mapping technology applicable in a daily clinical practice for identification of focal arrhythmia sources in the aforementioned cardiac regions.

Significant accuracy improvement of ECGI in the septal area is crucially important to increase its clinical value.

\section{Acknowledgements}

We would like to express our sincere thanks and deepest appreciation to Dr. Andrey Kozlenok, the Head of Department of Cardiovascular Physiology for his great support in organizing and hosting of this study.

\section{References}

[1] J. L. Sapp, F. Dawoud, J. C. Clements, and B. M. Horácek, "Inverse solution mapping of epicardial potentials: quantitative comparison with epicardial contact mapping," Circ Arrhythm Electrophysiol, vol. 5, no. 5, pp. 10011009, 2012.

[2] P. Oosterhoff et al., "Experimental Validation of Noninvasive Epicardial and Endocardial Activation Imaging," Circ Arrhythm Electrophysiol, vol. 9, no. 8, e004104, 2016.

[3] M. Namdar, M. Chmelevsky, A.-L. Hachulla, D. C. Shah, "Precise Noninvasive ECG Mapping Derived Localization of the Origin of an Epicardial Ventricular Tachycardia," Circ Arrhythm Electrophysiol, vol. 9, no. 12, 2016.

[4] L. R. Bear et al., "How Accurate Is Inverse Electrocardiographic Mapping?,” Circ Arrhythm Electrophysiol, vol. 11, no. 5, e006108, 2018.

[5] M. J.M. Cluitmans et al., "In Vivo Validation of Electrocardiographic Imaging,” JACC: Clinical Electrophysiology, vol. 3, no. 3, pp. 232-242, 2017.

Address for correspondence:

Mikhail Chmelevsky

Almazov National Medical Research Center,

Department of Electrocardiology,

2 Akkuratova street, Saint-Petersburg, 197341, Russia

boxmch@gmail.com 